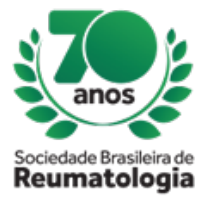

\title{
ERYTHROMELALGIA: A DIFFERENTIAL DIAGNOSIS OF CHRONIC PAIN
}

Raissa Velasques de Figueiredo (Universidade Federal de Ciências da Saúde de Porto Alegre , Porto Alegre, RS, Brasil), Maria Lúcia Lemos Lopes (Universidade Federal de Ciências da Saúde de Porto Alegre , Portp Alegre, RS, Brasil), Tatiana Freitas Tourinho (Universidade Federal de Ciências da Saúde de Porto Alegre, Porto Alegre, RS, Brasil), Maria Odete Esteves Hilário (Universidade Federal de Ciências da Saúde de Porto Alegre , Porto Alegre, RS, Brasil), Gilberto Scanagatta (Universidade Federal de Ciências da Saúde de Porto Alegre, Porto Alegre, RS, Brasil), Rafael Coradin (Universidade Federal de Ciências da Saúde de Porto Alegre, Porto Alegre, RS, Brasil), Luana Ribeiro Carlos (Universidade Federal de Ciências da Saúde de Porto Alegre, Porto Alegre, RS, Brasil), Thiago Willers (Universidade Federal de Ciências da Saúde de Porto Alegre, Porto Alegre, RS, Brasil), Raquel dos Santos Ramos (Universidade Federal de Ciências da Saúde de Porto Alegre, Porto Alegre, RS, Brasil), Tatiane Andressa Gasparetto (Universidade Federal de Ciências da Saúde de Porto Alegre, Porto Alegre, RS, Brasil)

\section{BACKGROUND}

Erythromelalgia is characterized by intense burning pain, significant erythema and increased skin temperature. It is more common that it affects hands and feet, but can also affect ears and nose. Exposure to heat is the trigger of symptoms, while exposure to cold causes relief. The symptoms are usually bilaterally, but may even occur unilaterally or predominantly on one side.

\section{CASE REPORT}

A 23-year-old white patient with type I glycogenosis with painful burning, heat and intense blushing of the hands and feet. The condition had started two years ago, but worsening about one month ago. Symptoms worsened with the heat, being necessary frequent cooling of the extremities. There were periods of spontaneous improvement of symptoms. Physical examination showed flushing in feet and hands. There was no trauma history, arthritis, signs of secondary infection, fever or inappetence. Treatment with corticosteroids, gabapentin, duloxetine, NSAIDs and hydroxychloroquine had already been done without improvement of symptoms.

During the hospitalization, a skin biopsy was performed but it was inconclusive, exams that excluded active rheumatic diseases and infections were performed.

\section{CONCLUSION}

This is a secundary form of Erythromelalgia, the primary form is rare and autosomal dominant. There should always be a suspicion that there may be bone marrow involvement. Skin biopsy is usually inconclusive. The evaluation with electroneuromyography is necessary to rule out differential diagnoses.

The symptoms can last hours and the intensity of the pain may be disproportionate to the clinical finding. The symptoms contribute to a worsening of the quality of life of the patient. Other skin changes such as Raynaud's Phenomenon, erythema pernium and livedo reticular may occur concurrently.

The pathophysiology of the disease involves both adrenergic alterations such as sweating and neuropathy of fine fibers, alterations of the sweat glands would be related to the activation of $C$ fibers contributing to the pain picture.

It is an important differential diagnosis among other diseases that may cause chronic extremity pain such as trauma, infection, antiphospholipid antibody syndrome, Raynaud's purpura phase, vasculitis, thrombophlebitis obliterans, atherosclerosis obliterans, acrocyanosis, Fabry's disease, paraneoplasia. 
The control of the underlying disease is the ideal treatment, to control the symptoms should avoid exposure to heat, physical activity, smoking, stress. The use of aspirin shows partial improvement, the use of tricyclic antidepressants and sodium channel blockers are not effective, the use of topical capsaicin cream has been beneficial. The use of anesthetic blockade of nerve plexuses may be necessary. 